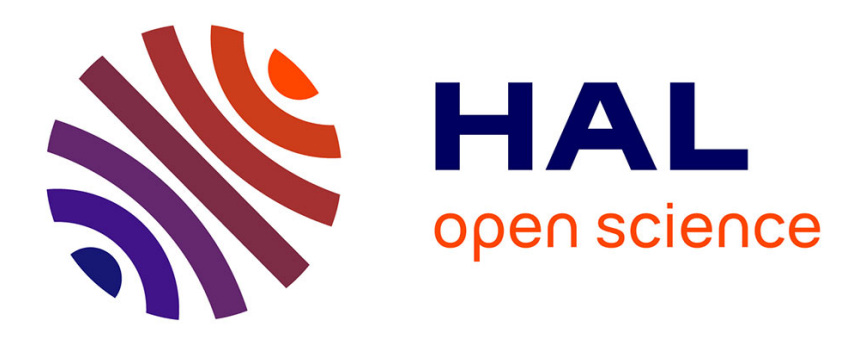

\title{
Sublimation-driven morphogenesis of Zen stones on ice surfaces
}

\author{
Nicolas Taberlet, Nicolas Plihon
}

\section{To cite this version:}

Nicolas Taberlet, Nicolas Plihon. Sublimation-driven morphogenesis of Zen stones on ice surfaces. Proceedings of the National Academy of Sciences of the United States of America, 2021, 118 (40), pp.e2109107118. 10.1073/pnas.2109107118 . hal-03419327

\section{HAL Id: hal-03419327 \\ https://hal.science/hal-03419327}

Submitted on 8 Nov 2021

HAL is a multi-disciplinary open access archive for the deposit and dissemination of scientific research documents, whether they are published or not. The documents may come from teaching and research institutions in France or abroad, or from public or private research centers.
L'archive ouverte pluridisciplinaire HAL, est destinée au dépôt et à la diffusion de documents scientifiques de niveau recherche, publiés ou non, émanant des établissements d'enseignement et de recherche français ou étrangers, des laboratoires publics ou privés. 


\title{
Sublimation-driven morphogenesis of Zen stones on ice surfaces
}

\author{
Nicolas Taberlet ${ }^{\mathrm{a}, 1}$ and Nicolas Plihon ${ }^{\mathrm{a}}$ \\ a Univ Lyon, Ens de Lyon, Univ Claude Bernard, CNRS, Laboratoire de Physique, F-69342 Lyon, France \\ This manuscript was compiled on November 8, 2021
}

In this article, the formation of Zen stones on frozen lakes and the shape of the resulting pedestal are elucidated. Zen stones are natural structures in which a stone, initially resting on an ice surface, ends up balanced atop a narrow ice pedestal. We provide the first physical explanation for their formation, sometimes believed to be caused by the melting of the ice. Instead, we show that slow surface sublimation is indeed the physical mechanism responsible for the differential ablation. Far from the stone the sublimation rate is governed by the diffuse sunlight whilst in its vicinity, the shade it creates inhibits the sublimation process. We reproduced the phenomenon in laboratory-scale experiments conducted in a lyophilizer, and study the dynamics of the morphogenesis. In this apparatus, which imposes controlled constant sublimation rate, a variety of model stones consisting of metal disks was used, which allows us to rule out the possible influence of the thermal conduction in the morphogenesis process. Instead we show that the stone only acts as an umbrella whose shade hinders the sublimation, hence protecting the ice underneath which leads to the formation of the pedestal. Numerical simulations, in which the local ablation rate of the surface depends solely on the visible portion of the sky, allow us to study the influence of the shape of the stone on the formation of the ice foot. Finally, we show that the far-infrared black-body irradiance of the stone itself leads to the formation of a depression surrounding the pedestal.

Morphogenesis | Differential Ablation | Sublimation

A wide variety of spectacular structures in which a stone or rock sits on a slender pedestal can be found in Nature: hoodoos consist of a hard stone protecting a narrow column of sedimentary rock from rain-induced erosion (1-3), mushroom rocks whose base is eroded by strong particle-laden winds (4), glacier tables for which a foot of ice resists melting due mostly to thermal insulation provided by a large rock (5-8) *, and Zen stones on frozen lakes in which pebbles rest on delicate ice pedestals, as shown in Fig. 1a) and b). Although these structures differ in the nature of the erosion mechanisms, in time-scales (from days for Zen stones to centuries for hoodoos), and in size dimension (from centimetres to tens of meters), the resulting shapes can be strikingly similar. Note also that micro and nano-fabrication processes, largely based upon differential etching of metal or semiconductor substrates, may lead to the formation of micrometer-sized opto-mechanical resonators with a geometry very similar to that of Zen-stones $(9,10)$. Most natural structures in which a stone sits on a pedestal are due to differential melting or mechanical abrasion. However, ice sublimation is known to drive a wide variety of morphologies in astrophysical bodies $(11,12)$. On Earth, sublimation-driven morphologies are rather scarce, one wellknown example being penitentes (13-15) encountered in the

* The similarities and differences between glacier tables and Zen-stones are discussed in the conclusion

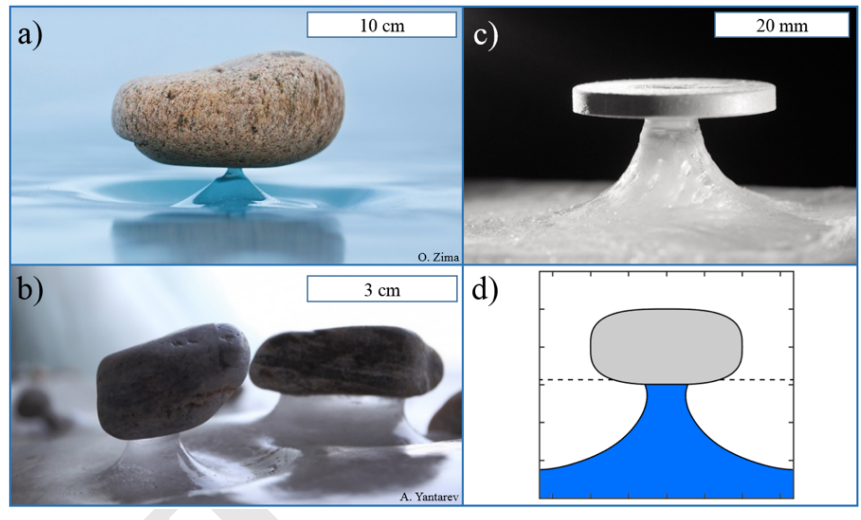

Fig. 1. Zen stones in Nature, in the lab, and in numerical simulations. a) Zen stone on Lake Baikal (approximately 16-cm-wide) showing a narrow ice pedestal. b) Zen stones (approximately 3 to $5 \mathrm{~cm}$-wide) in a cave (Lake Baikal) with no direct sunlight. c) Laboratory-scale experiment using a $30-\mathrm{mm}$ aluminium disk initially resting on a flat ice surface and placed in a lyophilizer during 40 hours. d) 2D numerical simulation of the phenomenon (see text for details).

Andes and the Himalayas. However, numerous observations have been made throughout the Solar System, ranging from penitentes on Pluto (16), to landscape formation on Mars (1719), Pluto (20), (1)Ceres (21), satellites of Jupiter (22-24), Saturn (25) and comets $(26,27)$.

In this article, we show that the morphogenenis of Zen stones is governed by differential sublimation rates of ice due to the shade provided by the stone, thus reporting a new natural occurence of sublimation-driven pattern formation

\section{Significance Statement}

Zen stones are fascinating natural structures consisting in a stone standing on a slender ice pedestal, whose origin had long been misunderstood. We demonstrate that they are caused by a variation in the sublimation rate of the surrounding ice, leading to the slow formation of a pedestal, adding to the very few reports of sublimation driven pattern formation. Understanding this process sheds new light on other differential ablation processes encountered on ice surfaces, such as debris-covered glaciers, whose existence is threatened by global warming, and icy bodies in space. Indeed, NASA's Europa Lander project aims to seek biosignatures on Jupiter's ice-covered moon on the surface of which differential sublimation may threaten the lander stability and needs to be fully understood.

N.T. developed and ran the numerical simulations. N.T. performed experiments with the assistance of N.P.. All authors analyzed the results, and wrote the article.

The authors declare no competing interests.

${ }^{1}$ To whom correspondence should be addressed. E-mail: nicolas.taberlet@ens-lyon.fr 
on Earth. The Zen stones are reproduced in the laboratory (see Fig. 1c) using an apparatus which allows for a controlled constant sublimation rate, whilst numerical simulations based on a geometrical numerical model recover the experimental results and allows us to study the influence of the shape of the stone on the formation of the ice pedestal (see Fig. 1d).

\section{Zen stones in Nature}

Zen stones on ice surfaces consist in natural phenomena in which a stone sits at the top of a slender ice pedestal (Fig. $1 \mathrm{a}-\mathrm{b}^{\dagger}$ ). Initially, the stone rests directly on a flat ice surface but as the frozen surface is gradually eroded, the ice under the stone is protected by a mechanism that remained unexplained to date. Over time, the stone remains at its original altitude as the differential ablation induced by the umbrella effect carves an increasingly taller and thinner foot in the ice below, eventually causing the stone to fall off. Zen stones are rare in Nature and predominantly found in the Small Sea of Lake Baikal, Russia, which is frozen for an averaged 5 months/year and where the ice layer typically reaches $1 \mathrm{~m}$ at the end of winter (28). The scarcity of the phenomenon stems from the rarity of thick flat snow-free layers of ice, which require longstanding cold and dry weather conditions. Weather records show that melting of the ice is virtually impossible and that instead, the weather conditions (wind, temperature and relative humidity) favor sublimation (SI Fig.1 and 2) which has long been known to be characteristic of the Lake Baikal area (28). In particular, over a time interval spanning 7 weeks prior to the date the photograph shown in Fig. 1a) was taken, temperatures remained below $0^{\circ} \mathrm{C}$, with an average daily maximum of $-15.3^{\circ} \mathrm{C}$, and an averaged daily maximum relative humidity of $83 \%$. From the average winter solar irradiance at the Small Sea and the latent heat of sublimation of water, one can estimate the ablation rate of an ice surface $u \sim 2 \mathrm{~mm} /$ day, which gives a characteristic formation time of the pedestal $W / u \sim 40$ days for the stone of Fig. 1a (where $W$ is the half-width of the stone, typically $8 \mathrm{~cm}$ in Fig. 1a). Sublimation is thus a slow surface process, as well as an endothermic phase transition which requires a constant flux of external energy, provided in Nature by solar irradiance $(11,13,14)$. Indeed, a net energy input of $60 \mathrm{~W} / \mathrm{m}^{2}$ (or $5 \mathrm{MJ} / \mathrm{m}^{2} /$ day) is required for the ice to sublimated (far from the stone) at the rate of $2 \mathrm{~mm} /$ day. At the latitude of Lake Baikal, in February or March, the irradiance reaches from 400 to $500 \mathrm{~W} / \mathrm{m} 2$ for typically 6 to 8 hours. Assuming an emissivity of 0.95 , the ice itself (at 260K) radiates $246 \mathrm{~W} / \mathrm{m} 2$ back into the atmosphere. During the day, the net energy flux therefore largely exceeds what is need to sublimate a few $\mathrm{mm}$ of ice while the process stops at night. Note that this sublimation rate is only an average estimate and that the actual rate may depends on the exact optical properties of the ice (in particular multiple scattering in white ice which can considerably affect the energy balance (29)) as well as on fluctuation weather conditions. Regarding the energy balance of the stone itself, a pebble placed on the ice surface will receive the sunlight in place of the patch of ice it covers, possibly causing it to heap up. However, due to the good thermal contact with the ice, to the heat exchange with the cold ambient windy air and to the far-infrared radiation it emits back into the atmosphere, the temperature of the stone

\footnotetext{
$\dagger$ The photograph in Fig. 1a was taken by O. Zima (instagram.com/zima landscape), in Fig 1b by A.
} Yanarev and in Fig. 4e by S. Tolstnev (facebook.com/tolstnev), all in the Small Sea of Lake Baikal. will remain very close to that of the ice. Therefore, the energy captured by the stone creates a deficit in the energy received by the ice. In simple terms, the stone acts as an umbrella under which the sublimation rate is greatly decreased.

One remarkable aspect of the ice pedestals is their axisymmetric shape, which might appear counter-intuitive given that the position of the sun in the sky is preferentially oriented $\left(20^{\circ}\right.$ above horizon and $\pm 60^{\circ}$ from the South in the winter). This apparent contradiction is explained by the typical cloud cover during winter which scatters sunlight, rendering the irradiance more isotropic. The irradiance is maximum at the zenith (i.e. in the vertical direction), regardless of the actual position of the sun (SI Fig. 3), and the luminous flux should be more isotropic than highly directional. Finally, although ablation appears to be caused be sublimation, it remains important to rule out other plausible mechanisms. Firstly, direct sunlight might overheat the stone. However, the heat diffusion time of stones (computed from their size and thermal diffusivity) is of the order of several minutes, which implies that any overheating would quickly diffuse to the ice, causing it to melt rather than forming a pedestal. Secondly, wind-driven snow particle are known to cause mechanical erosion (30) but the clear and smooth natural pedestals show no indication of mechanical wear, and the typical time-scale of wind-driven erosion is far too large. Thirdly, the melting temperature of water is known to vary with the ambient pressure. However, the phase-diagram of water (SI Fig. 2) reveals that it changes significant only for pressures above $10^{7} \mathrm{~Pa}$. The additional pressure of Zen stones on the ice does not exceed $10^{5} \mathrm{~Pa}$, far below the point where it could cause melting of the ice (which anyway would not create a pedestal). Finally, ice is known to creep (i.e. undergo a slow plastic deformation) under high pressure (31). However, at $-20^{\circ} \mathrm{C}$ the typical deformation rate under a pressure of $10^{5} \mathrm{~Pa}$ is only $10^{-9} \mathrm{~s}^{-1}$, corresponding to a typical far-too-large deformation time of years. Note however that when a stone is initially placed on the ice surface, the stress would be locally high at the few points where the stone touches the ice. Given the high homologous temperature $T_{\text {ice }} / T_{\text {melting }} \simeq 0.95$ grain boundary migration $(32,33)$ could certainly occur initially causing the ice to creep and the stone to slightly settle into the ice. As the actual surface of contact between the stone and the ice increases, the stress strongly decreases and any deformation rapidly stops.

\section{Laboratory experiments}

We have reproduced the formation of Zen stones in a laboratory-scale experimental setup. A metal disk (of radius $W=15 \mathrm{~mm}$ ) representing the stone is placed at the surface of a block of ice sublimating in the chamber of a commercial lyophilizer (see Methods). The external energy required to sublimate the ice originates from the infrared (IR) black-body radiation of the outer walls of the vacuum chamber, which remain at room temperature (see Fig. 2a). The ablation is then isotropic (in the absence of a stone), and mimics the relative isotropy of natural diffuse sunlight in overcast weather, with a sublimation rate of typically $8-10 \mathrm{~mm}$ /day (see Methods).

Figure 1c shows an artificial Zen stone obtained using a aluminium disk, initially resting on the ice surface, after $40 \mathrm{~h}$ in the lyophilizer, whilst Fig. 2b-d) shows the experimental results using three different metal disk s (SI Video 1 shows a time-lapse of the experiment displayed in Fig. 2d).). Clearly, 

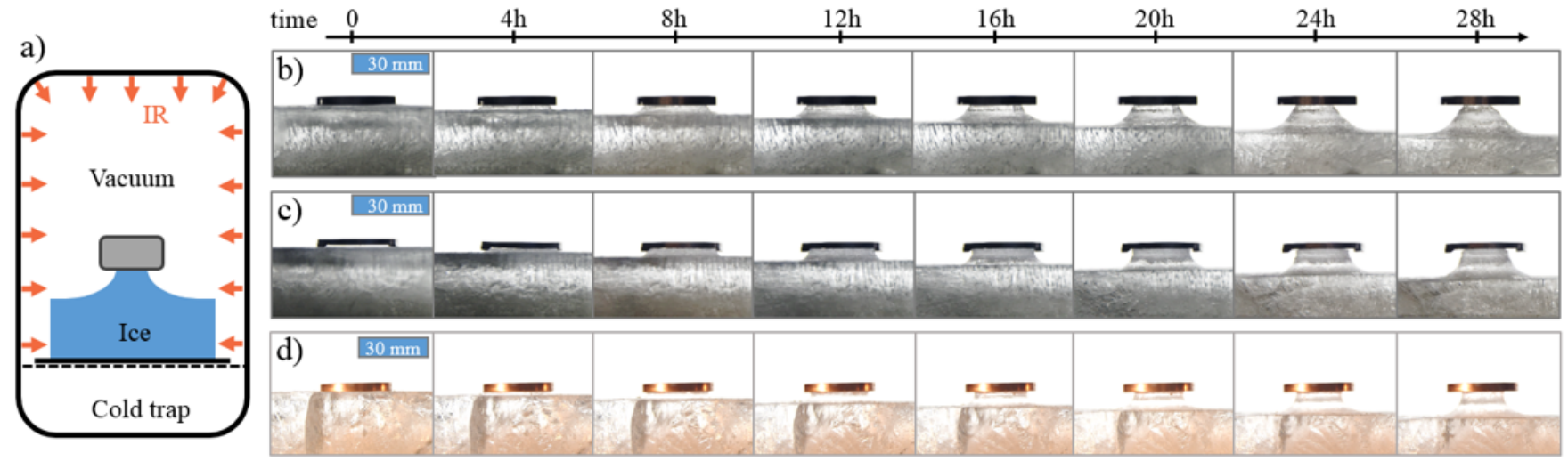

Fig. 2. Laboratory-scale formation of Zen stones on ice. a) Sketch of the experimental setup. A metal disk rests on an initially flat block of ice in a lyophilizer. The energy required for the sublimation originates from the black-body radiation (in the far-infrared) of the vacuum chamber. Image sequence using b) a $30 \mathrm{~mm}$ aluminium disk, $\mathrm{c}$ ) a grooved aluminium disk which induces poor thermal conduction with the ice and d) a 30-mm copper disk.

our simplified setup is capable of qualitatively reproducing the formation of natural Zen stones. The evolution is shown over the first $28 \mathrm{~h}$ of operation since for longer times, the pedestals become too narrow and small asymmetries as well as mechanical vibrations often cause the disk to fall off. In Nature, the process is slow (several weeks), which ensures a good thermal equilibrium of the stone. In our setup however, due to the quasi-absence of air in the chamber, the stone can overheat (while remaining far below the melting temperature of ice). The excess energy accumulated in the stone then diffuses to the ice directly in contact with the stone, causing it to sublimate (SI Fig. 4). In order to minimize this inconvenient side-effect, thin metal disks made of polished metal were used, with higher reflectivity to IR radiations as compared to unpolished surface. Fig. $2 \mathrm{~b}$ and $2 \mathrm{~d}$ show Zen stones obtained in the laboratory using aluminum and copper disks. The dynamics and the morphology of the two pedestals are very similar, although the thermal conductivity of $\mathrm{Cu}$ is nearly twice as large as that of $\mathrm{Al}$. This result indicates that the thermal properties of the material are not critical for the formation of Zen stones, and that the main mechanism is the lack of radiation that the stone causes in its vicinity, leading to differential ice sublimation. Fig. 2c uses an aluminum disk, initially identical to that of Fig. 2b, in which a central groove is milled. Initially, the surface of contact between the disk and the ice is therefore extremely reduced, which limits heat conduction. Although the exact morphologies of the pedestals are somewhat different, their overall shapes are very similar. The sole fact that a well-formed pedestal appears for the grooved disk is in itself an important result. Indeed, one plausible cause of differential ablation could have been that the sublimation was blocked by mechanical contact with the stone, in the same way a thin plastic sheet can prevent a surface of water from evaporating. Instead, the milled-disk experiment indicates again that the stone act as an umbrella, shielding the ice from external radiations (diffuse sunlight in Nature and infrared in the lyophilizer), therefore hindering the sublimation in its shade.

It is worth mentioning that two preparation protocols were used. In the original procedure, the "stone" was simply placed on the ice surface, whereas in a second protocol the stone was initially embedded within the ice (when the ice block was built). In the former case, there were only initially a very few contact points with the ice and deformation could certainly occur. In the latter case however, the weight of the stone was always even distributed and the stress remained very low (a few Pascals). Both protocols lead to identical shapes for the pedestal, showing that over the long term, ice deformation or grain boundary diffusion does not play a role. Note that in theory, in latter stages when the pedestal becomes very narrow, the pressure exerted by the stone should diverge, possibly causing the ice to creep. However, as mentioned above, small perturbations and irregularities in the shape (both in the field and in our experiments) always caused the stone to falls off from its pedestal before this creep regime is ever reached.

\section{Numerical modelling}

We have reproduced the formation of Zen stones in numerical simulations. As explained above, ablation is a surface process and we model the surface of the ice as a series of connected points. For simplicity, the diffuse light is assumed to be isotropic and the local luminous flux is proportional to the angle $\phi$ that the sky subtends at a given point (Fig. 3a). Our simulations compute the evolution of a $2 \mathrm{D}$ ice surface hypothesizing that the direction of sublimation is locally normal to the surface and that the rate of sublimation depends on the external radiative energy received (the computation of which is straightforward). The surface is simulated by an array of $n$ points $M_{n}\left(x_{n}, y_{n}\right)$ at time $t$, whose position at time $t+d t$ is computed (see Fig. 3b). The half-width of the stone is set to $W=1$ and the velocity of the sublimation front in the absence of the stone (i.e. for $\phi=180^{\circ}$ ) is set to $u=1$, but the choice of these numerical values has no effect on the resulting shape since our simulation rely on purely geometrical laws. The characteristic formation time $\tau=W / u$ is used as a scale for the computational time, and the integration time-step $\mathrm{d} t$ is set to $10^{-4} \tau$.

Initially the surface is flat, the first point is in contact with the stone and each point is placed at a distance chosen to be $1 / 2000$ th stone half-width. Due to the simple mathematical form chosen for the stone, its bottom is always curved and never truly flat. Therefore, in our numerical model the stone is initially nested within the ice. The distance between the position of the nth point at times $t$ and $t+d t$ is given by $d l_{n}=u d t \phi_{n} / 180^{\circ}$. The direction of the displacement $d \vec{l}_{n}$ is chosen to be perpendicular to the two closest neighboring 


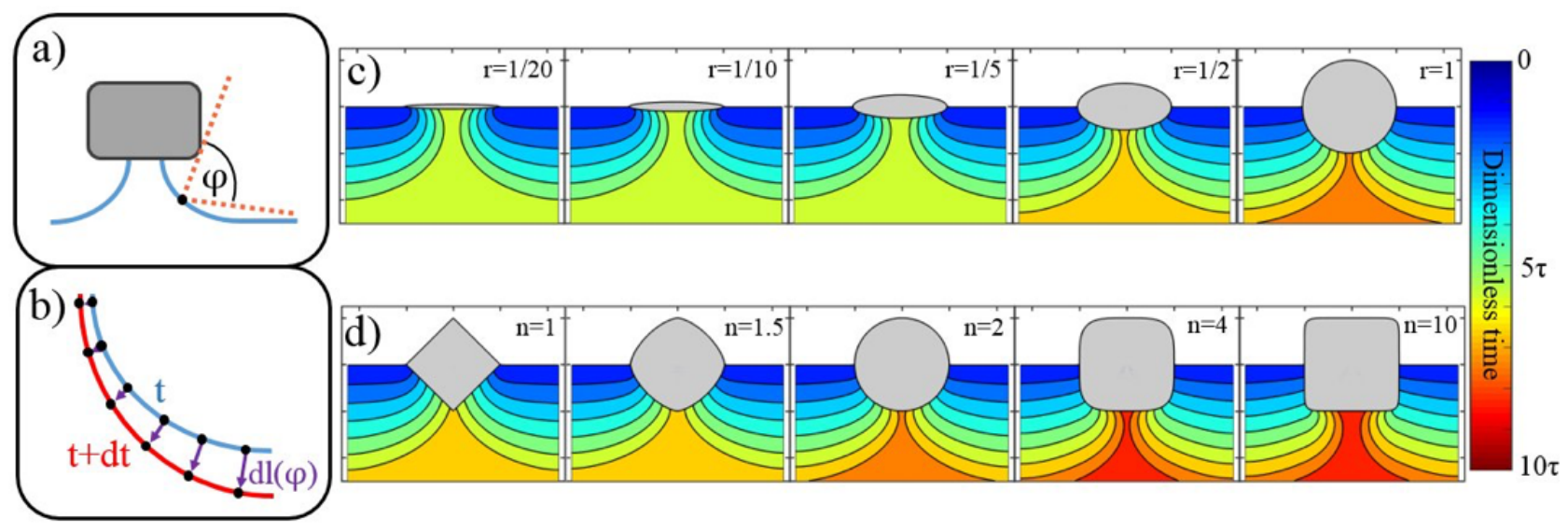

Fig. 3. 2D numerical modelling of Zen stones. a) Sketch showing the portion of sky, $\phi$ visible from a position at the surface of the ice. b) Sketch of the surface at two consecutive time-steps. The ablation rate of the surface depends on the angle $\phi$ and the sublimation is locally perpendicular to the surface. c) Formation process of the pedestal for ellipses of varying height-to-width ratio $r$. The colour scale indicates the dimensionless elapsed time from the initial flat surface. d) Effect of the shape of the simulated stone on the morphology of the pedestal, for an aspect ratio of 1 .

points, i.e. to the $\left(M_{n-1} M_{n+1}\right)$ segment. This procedure cannot be applied to the first point of the surface $M_{1}$, which is by definition in contact with the stone. Instead, the location of $M_{1}$ is always given by the point where the $\left(M_{2} M_{3}\right)$ line intersects with the stone. Depending on the local slopes of the stones and the surface, it can happen that $\overrightarrow{d l}_{2}$ is such that $M_{2}(t+d t)$ rests within the stone. In that case, the surface is remeshed by simply relabeling the $\mathrm{n}^{\text {th }}$ point into $\mathrm{n}-1$, and by removing the first point $M_{1}$.

This morphogenesis model therefore relies on geometrical arguments (inspired by the experimental results in the lyophilizer) rather than on overly complex thermodynamic processes, and is intended to qualitatively reproduce the mechanisms. The shape of the stone is given by: $\left(\frac{x}{W}\right)^{n}+\left(\frac{y}{H}\right)^{n}=$ 1, where $W$ and $H$ are the half-width and half-height, and $n$ an exponent that determines the shape. Fig. 3c shows the temporal evolution of the ice surface for elliptical $(n=2)$ stones of varying aspect ratio $r=W / H$ whilst Fig. 3d shows the results obtained for stones of various shapes with an aspect ratio of 1 . As expected, the dynamics of the formation of the pedestal depends on the morphology of the stone. In all cases, a flat base produces an arched pedestal (see Fig. 3c $r=1 / 20$ or Fig. $3 \mathrm{~d} n=10$ ), which corresponds to empirical observations in Nature and in our model experiments, whereas a rounder base (see Fig. 3c $r=1$ or Fig. $3 \mathrm{~d} n=1.5$ ) leads to moderate slopes. Moreover, the time required for the pedestal to reach the center of the stone (i.e. the life time of the Zen stone) varies with the exact shape of the stone. This simple numerical model thus shows that the shade provided by the stone provides favorable conditions for differential sublimation and leads to the formation of Zen stones for a variety of shapes.

\section{Depression surrounding the pedestal}

There is yet another striking feature of Zen stones that remains to be explained: the pedestal is always surrounded by a more or less pronounced depression (or dip or basin). This dip is clearly visible in Fig. 1a, somewhat less marked on the leftmost stone in Fig. 1b, and particularly evident in Fig. 4e where its shape manifestly follows that of the stone. In all observable cases the dip is slightly larger than the typical size of the stone, whilst its exact morphology is clearly influenced by the shape of the stone. The very existence of a dip, relative to the average ice-surface, indicates that the sublimation rate must be locally greater than that induced by the diffuse sunlight alone. The additional energy causing this local increase in the ablation rate can be attributed to the black-body radiation of the stone itself which emits far infrared radiations (FIR), its temperature being in the in the range of $-20^{\circ} \mathrm{C}$ to $0^{\circ} \mathrm{C}$. While the umbrella effect is dominant regarding the overall dynamics of Zen-stones, the FIR emitted by the stone is a necessary secondary effect which creates the depression surrounding the pedestal. Fig. 4a shows a schematic of this mechanism and Fig. $4 \mathrm{~b}$ sketches the typical energy profiles of diffuse sunlight (blue) and of FIR (red). Note that the irradiance of the sun in typically 100 times larger than that of the stone (Fig. 4c), but the values of the integrated energies are comparable ( 359 $\mathrm{W} / \mathrm{m}^{2}$ for the solar energy vs. $259 \mathrm{~W} / \mathrm{m}^{2}$ from the stone at 260 $\mathrm{K})$. Moreover, the range of wave-lengths differ strongly: 300$2500 \mathrm{~nm}$ for sunlight vs. 5-50 $\mu \mathrm{m}$ for FIR. This difference has crucial implications since the extinction rate, i.e. imaginary part of the refraction index, varies over 10 decades (Fig. 4d). Similarly, the absorption length of ice strongly depends on the wavelength (Fig. 4d): $10 \mathrm{~km}$ for a $400-\mathrm{nm}$ radiation, meaning that the ice layer is then nearly transparent, vs. $10^{-5} \mathrm{~m}$ for a $10-\mu \mathrm{m}$ radiation, meaning that the energy is absorbed within a $10-\mu$ m-thick layer, which facilitates surface sublimation of the ice. Note that the data in Fig. 4d corresponds to pure ice and although multiple scattering in white ice may change the exact values of the extinction rate, the disparity remains huge and the conclusions still hold. This colossal disparity implies that even if the FIR irradiance is two orders of magnitude lower than that of the diffuse sunlight, it can locally significantly increase the ablation rate. This explains why the depression mirrors the shape of the stone (Fig. 4e). Note also how the dip is deeper on the right-side of the stone in Fig. 1b, due to more favourable orientation of the face on that side. The energy balance is therefore very subtle and varies over the during of a day since the temperature of the stone and obviously the amount of solar energy change from day to night. This is reminiscent of ablation processes of snow in forests for which the ablation rate can be either greater or less than surrounding 
a)

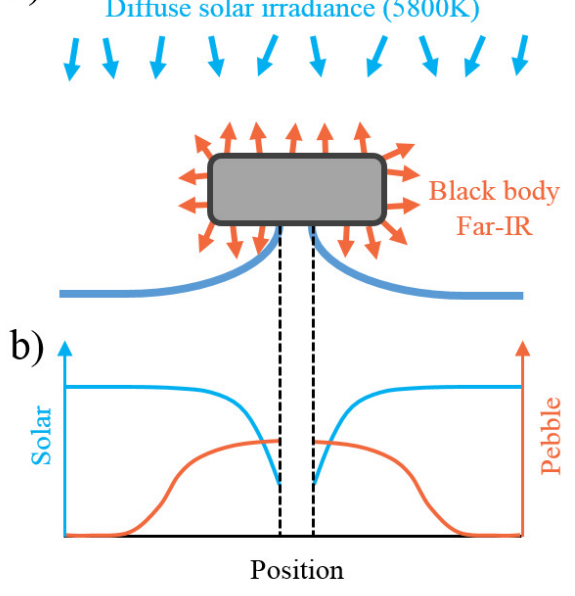

c)

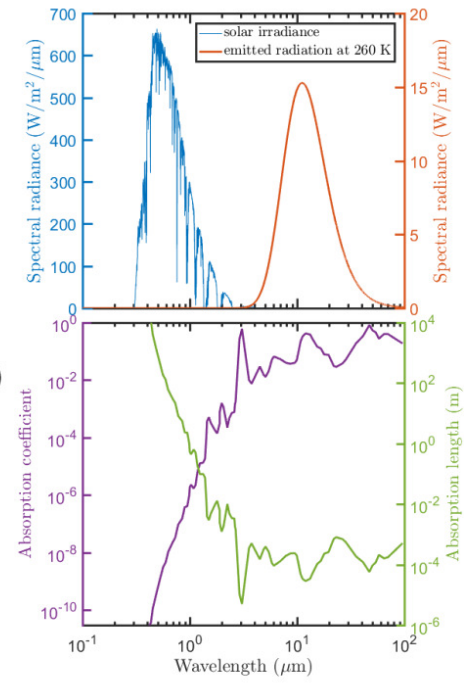

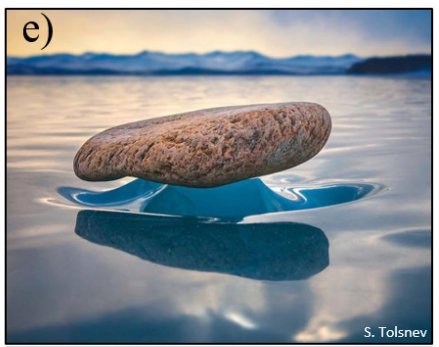

f)

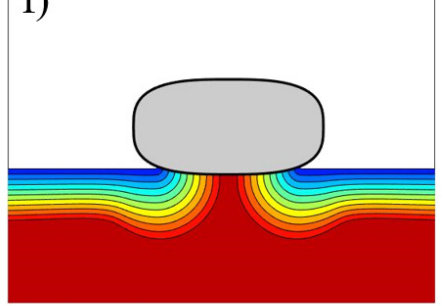

Fig. 4. Formation of the depression surrounding the ice pedestal. a) Sketch showing the diffuse sunlight received by the ice and the black-body emission of the stone (of typical negative temperature close to $0^{\circ} \mathrm{C}$ ) in the far-infrared spectrum. b) Sketch on the energy received per unit surface of the ice from diffuse sunlight (blue) and black-body radiation of the stone (orange). c) Power spectrum of the solar irradiance at lake Baikal on February 1 at solar noon, generated using the SMARTS (34) (blue) and of the near-IR black body emission from the stone at $260 \mathrm{~K}$ (orange). d) Absorption coefficient (i.e. imaginary part of the refraction index) (purple) and absorption length (green) of pure ice (35-37) . e) Zen stone on Lake Baikal showing that a depression which follows the shape on an irregular stone. f) Numerical modelling which includes the effect of the black-body emission leading to a dip around the pedestal.

open areas (38).

The additional effect of the far-IR radiation emitted by the stone can be implemented in the numerical simulations by adding an incoming energy to the external luminous flux (from the simulated sky). This added energy needs to be constant under a flat stone and should vanish away from it. The added contribution to the sublimation velocity is modelled as follows:

$$
u_{I R}=u\left(1-\operatorname{erf} \frac{x-W}{L}\right)
$$

where $x$ is the horizontal direction (from the center of the stone) and $L=W / 5$ represents the characteristic length of IR illumination on the sides of the stone. As displayed in Fig. 4f), the added contribution in the FIR leads to the formation of a clear depression around the pedestal.

\section{Conclusion and discussion}

Model experiments and numerical simulations were shown to reproduce the spectacular morphogenesis of the ice pedestal of Zen stones observed on frozen lakes in Earth. We have demonstrated that the formation is driven by differential sublimation of the ice, caused by the shade in the infrared spectrum provided by the stone. A geometrical numerical model reproduces the experimental findings and helps study the influence of the shape of the stone. Finally we have shown that the dip surrounding the pedestal is caused by the far infrared radiations emitted by the stone itself, which enhance the overall sublimation rate in its vicinity.

As mentioned in the introduction very similar structures, known as glacier tables, may appear on low-altitude glaciers, where a rock initially sitting on the ice surface winds ups after a few days on top of an ice pedestal. These structures range from a few tens of centimeters up to a few meters. Although they look similar to Zen-stones, they are governed by different processes and their shape and dynamics differ strongly. Glacier tables appear on low-altitude glaciers where the weather conditions cause the ice to melt instead of to sublimate. They form in warm air while the ice remains at $0^{\circ} \mathrm{C}$, whereas Zen-stones form in air which is colder (typically $-30^{\circ} \mathrm{C}$ to $-10^{\circ} \mathrm{C}$ ) than the ice (itself below $0{ }^{\circ} \mathrm{C}$ ).

Depending on their size and material as well as on the weather conditions, a stone or a pile of sand may either locally increase the melting rate, causing it to sink into the ice, or instead hinder the melting process, leading to the formation of a glacier table or a dirt cone. In a recent study (8), some of us have shown that the differential melting of ice for glacier tables found on low-altitude alpine glaciers is dominantly caused by heat exchange with the surrounding air. The umbrella effect which dominate the formation of Zen-stones is therefore only secondary for glacier tables encountered in the Alps. Instead, under these conditions, glacier tables form because the rock may act as a thermal insulator and therefore, their thermal properties (conductivity and specific heat) are crucial. The conclusion is exactly opposite for Zen-stones as demonstrated by our experiments performed using a variety of materials that lead to the same shape. Moreover, since heat conduction within glacier tables is crucial, there exists a minimum thickness (which depends on the material) below which the stone sinks deep into the ice instead of forming a table. This regime cannot be observed in the conditions where Zen-stones form, and even a very thin stone will lead to an ice pedestal.

\section{Materials and Methods}

Experimental protocol. The ice samples are prepared using purified and degassed water poured into a rectangular plastic container $(15 \times 15 \mathrm{~cm}, 5 \mathrm{~cm}$ deep). A $5 \mathrm{~cm}$-thick block of Styrofoam is set to float at the surface of the water as it turns into ice (in a freezer at $-20^{\circ} \mathrm{C}$ ) in order to force the freezing front to travel from the bottom to the top, which helps avoiding the formation of cracks as the water expands. The metal disks (at $-20^{\circ} \mathrm{C}$ ) are "glued" on 
the surface of the ice blocks using a few drops of liquid water. In order to maximize the reflection of infrared radiations, the metal disks are polished using a buffing wheel. Ice samples are placed on a $1 \mathrm{~cm}$ thick Styrofoam plate on the bottom metal shelf of a commercial lyophilizer (Christ Alpha 1-2 LDplus) and the pressure is gradually decreased over the course of 30 minutes in order to avoid the formation of quenching cracks within the ice. Specifically, while ice samples can withstand a rapid initial decrease from 1 bar to 10 mbar, a further decrease down to the operating conditions of the lyophilizer (typically $50 \mu \mathrm{bar}$, or $5 \mathrm{~Pa}$ ) needs to be gradual.

Note that the temperature inside the lyophilizer depends note only on the pressure but also on the cooling power of the device and on its ability to condensate vapor in its cold trap. In the experiments reported here, the temperature was around $-45^{\circ} \mathrm{C}$, i.e. below the temperatures observed at Lake Baikal in winter 2016. The profiles of the ice surface were imaged using a Nikon D5600 digital camera placed $2 \mathrm{~m}$ away in order to minimize parallax. The experiment were illuminated using a LED panel (placed behind the vacuum chamber) and a regular lamp from the front, and the images are taken at regular 5-minute intervals.

Sublimation rate in the lyophilizer. In order to demonstrate that the sublimation rate is both constant in time and independent of the orientation of the ice surface, three blocks were simultenaously placed in the lyophilizer. Figure 5 shows that the sublimation rate remains constant over time (within error bars) and is identical for a vertical, horizontal and inclined surface, which indicates that the energy flux emitted by the surrounding vacuum chamber is isotropic and uniform. There is an initial spacing of $6 \mathrm{~mm}$ between the two first blocks, and of $12 \mathrm{~mm}$ between the second and third block For a narrow gap, the sublimation rate of the vertical surfaces is considerably reduced as the IR flux is hindered and for a wider gap, the effect is still clearly visible although less intense. Again, this shows that the source of energy required for the ice to sublimate is the black body radiation of the acrylic vacuum chamber which remains at room temperature.

The pressure within the chamber can be adjusted (typically in the $3 \mathrm{~Pa}-20 \mathrm{~Pa}$ range) which affects the sublimation rate. The data in Fig. 5 was obtained for $5 \mathrm{~Pa}$, and displays a typical sublimation rate of $8.5 \mathrm{~mm} /$ day.

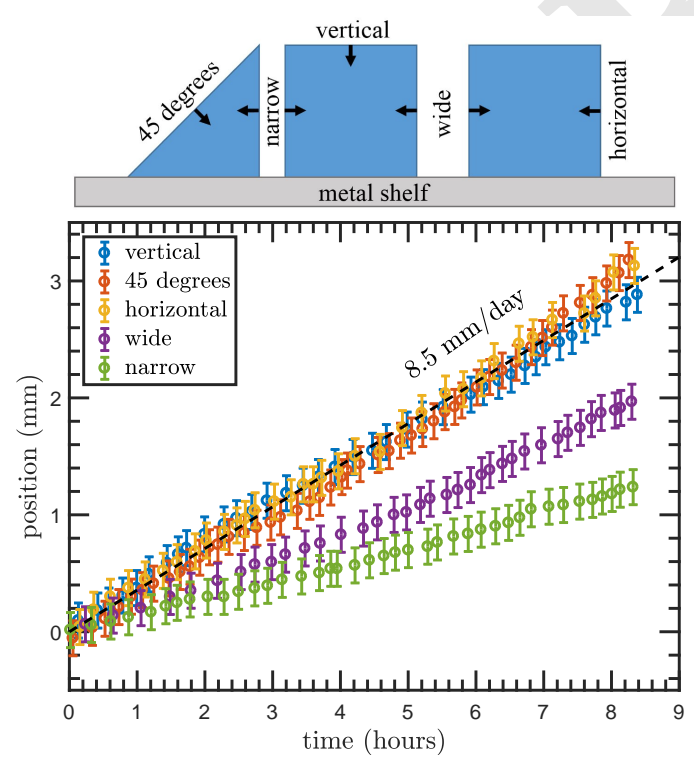

Fig. 5. Dynamic of sublimation of cubic blocks of ice (30 $\mathrm{mm}$ length) in the lyophilizer (sketch on top) measured from image processing.

ACKNOWLEDGMENTS. The authors are grateful to the Fédération de Recherche Marie André Ampère and to the Laboratoire de Physique at the ENS de Lyon for financial support.

1. Young R, Young A (1992) Sandstone Landforms, Lecture Notes in Physics. (Springer-Verlag).
2. Bruthans J, et al. (2014) Sandstone landforms shaped by negative feedback between stress and erosion. Nature Geoscience 7(8):597-601.

3. Turkington AV, Paradise TR (2005) Sandstone weathering: a century of research and innovation. Geomorphology 67(1-2):229-253.

4. Mashaal NM, Sallam ES, Khater TM (2020) Mushroom rock, inselberg, and butte desert landforms (gebel qatrani, egypt): evidence of wind erosion. International Journal of Earth Sciences 109(6):1975-1976.

5. Smiraglia C, Diolaiuti G (2011) Encyclopedia of Snow, Ice and Glaciers, eds. Singh VP, Singh P, Haritashya UK. (Springer), pp. 262-267.

6. Bouillette M (1933) Une superbe table des glaciers - in french. L'Astronomie 47:201-202.

7. Bouillette M (1934) La fin d'une table des glaciers - in french. L'Astronomie 48:89-91.

8. Hénot M, Plihon N, Taberlet N (2021) Onset of glacier tables. Physical Review Letters.

9. Min B, et al. (2009) High-q surface-plasmon-polariton whispering-gallery microcavity. Nature 457(7228):455-458.

10. Nguyen DT, et al. (2013) Ultrahigh q-frequency product for optomechanical disk resonators with a mechanical shield. Applied Physics Letters 103(24):241112.

11. Mangold N (2011) Ice sublimation as a geomorphic process: A planetary perspective. Geomorphology 126(1-2):1-17.

12. Law J, Dijk DV (1994) Sublimation as a geomorphic process: A review. Permafrost and Periglacial Processes 5(4):237-249.

13. Bergeron V, Berger C, Betterton MD (2006) Controlled irradiative formation of penitentes. Physical Review Letters 96(9).

14. Claudin P, Jarry H, Vignoles G, Plapp M, Andreotti B (2015) Physical processes causing the formation of penitentes. Physical Review E 92(3).

15. Betterton MD (2001) Theory of structure formation in snowfields motivated by penitentes, suncups, and dirt cones. Physical Review E 63(5).

16. Moores JE, Smith CL, Toigo AD, Guzewich SD (2017) Penitentes as the origin of the bladed terrain of tartarus dorsa on pluto. Nature 541(7636):188-190.

17. Head JW, Marchant DR (2003) Cold-based mountain glaciers on mars: Western arsia mons. Geology 31(7):641.

18. Massé M, et al. (2010) Martian polar and circum-polar sulfate-bearing deposits: Sublimation tills derived from the north polar cap. Icarus 209(2):434-451.

19. Keown LEM, Bourke MC, McElwaine JN (2017) Experiments on sublimating carbon dioxide ice and implications for contemporary surface processes on mars. Scientific Reports 7(1).

20. Moore JM, et al. (2017) Sublimation as a landform-shaping process on pluto. Icarus 287:320333

21. Nathues A, et al. (2015) Sublimation in bright spots on (1) ceres. Nature 528(7581):237-240.

22. Howard AD, Moore JM (2008) Sublimation-driven erosion on callisto: A landform simulation model test. Geophysical Research Letters 35(3).

23. Carlson RW, et al. (2009) Europa, eds. Pappalardo RT, McKinnon WB, Khuranaijay K. (University of Arizona Press, Tucson), pp. 283-327.

4. Team ELMC (2016) Europa lander mission, d-97667, (NASA JPL), Technical report.

25. Spencer JR, Denk T (2009) Formation of iapetus' extreme albedo dichotomy by exogenically triggered thermal ice migration. Science 327(5964):432-435.

26. Britt D, et al. (2004) The morphology and surface processes of comet $19 / p$ borrelly. Icarus 167(1):45-53

27. Thomas N, et al. (2015) The morphological diversity of comet $67 \mathrm{p} /$ churyumov-gerasimenko. Science 347(6220):aaa0440-aaa0440.

28. Livingstone DM (1999) Ice break-up on southern lake baikal and its relationship to local and regional air temperatures in siberia and to the north atlantic oscillation. Limnology and Oceanography 44(6): 1486-1497.

29. Mullen PC, Warren SG (1988) Theory of the optical properties of lake ice. Journal of Geophysical Research: Atmospheres 93(D7):8403-8414.

30. Dietrich R (1977) Wind erosion by snow. Journal of Glaciology 18(78):148-149.

31. Goodman DJ, Frost HJ, Ashby MF (1981) The plasticity of polycrystalline ice. Philosophical Magazine A 43(3):665-695.

32. Montagnat M, Duval $P(2000)$ Rate controlling processes in the creep of polar ice, influence of grain boundary migration associated with recrystallization. Earth and Planetary Science Letters 183(1-2):179-186.

33. Nasello O, Di Prinzio C, Guzmán P (2005) Temperature dependence of "pure" ice grain boundary mobility. Acta Materialia 53(18):4863-4869.

34. Gueymard CA (2019) The smarts spectral irradiance model after 25 years: New developments and validation of reference spectra. Solar Energy 187:233-253.

35. Warren SG, Brandt RE (2008) Optical constants of ice from the ultraviolet to the microwave: A revised compilation. Journal of Geophysical Research: Atmospheres 113(D14).

36. Picard G, Libois $Q$, Arnaud L (2016) Refinement of the ice absorption spectrum in the visible using radiance profile measurements in antarctic snow. The Cryosphere 10(6):2655-2672.

37. Warren SG (2019) Optical properties of ice and snow. Philosophical Transactions of the Royal Society A 377(2146):20180161.

38. Lundquist JD, Dickerson-Lange SE, Lutz JA, Cristea NC (2013) Lower forest density enhances snow retention in regions with warmer winters: A global framework developed from plot-scale observations and modeling. Water Resources Research 49(10):6356-6370. 


\section{Supplementary Information}
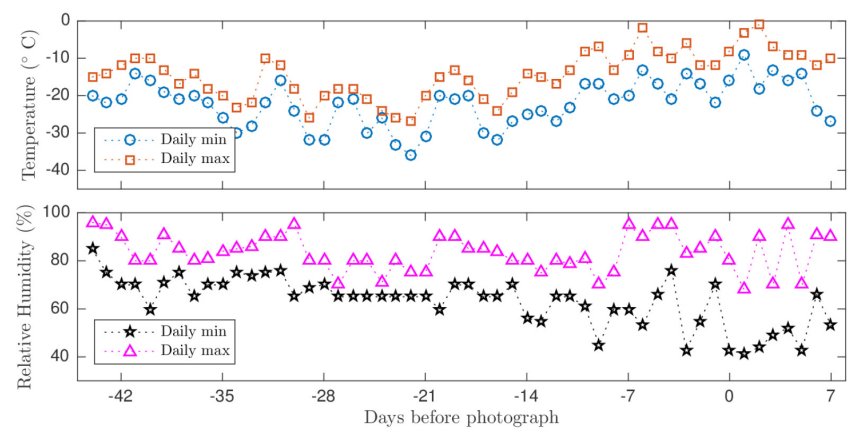

Fig S1. Meteorological records on the Small Sea of Lake Baikal over 8 weeks prior to the date picture 1a) was taken (on Feb. 12, 2016). The maximum temperature remains below freezing and the weather is often overcast. The relative humidity typically ranges from $40 \%$ to $100 \%$. Data retrieved from https://www.timeanddate.com/weather/@2022143/historic? month $=1 \&$ year $=2016$.

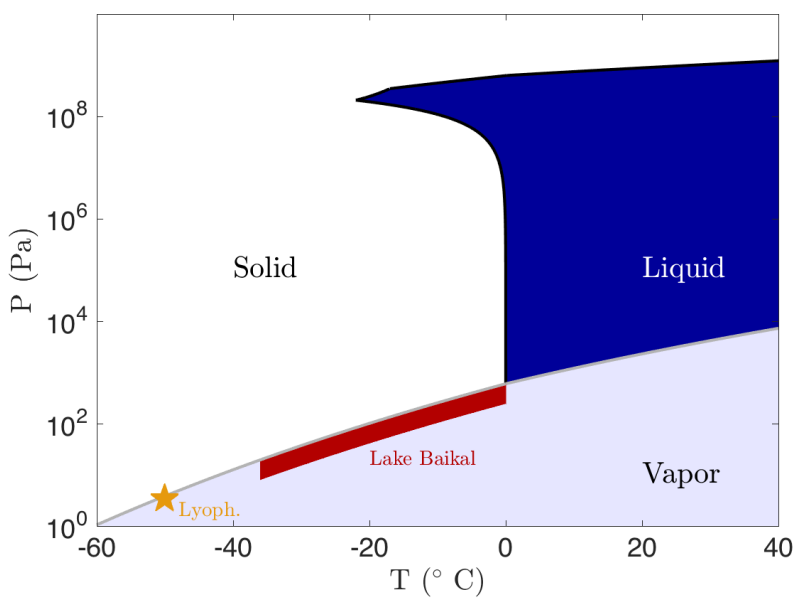

Fig S2. Phase diagram of pure water on a semi-logarithmic scale. The melting temperature decreases varies significantly only for pressures greater than $10 \mathrm{MPa}$, several orders of magnitude greater than that exerted by actual stones or by the metal disks used in this study. The star represents the typical operating conditions of the lyophilizer $\left(-50^{\circ} \mathrm{C}\right.$ and $\left.5 \mathrm{~Pa}\right)$ and the red region corresponds to the data of Supplementary Figure 1. Data taken from The International Association for the Properties of Water and Steam, Revised Release on the Pressure along the Melting and Sublimation Curves of Ordinary Water Substance, IAPWS R14-08 (2011), http://www.iapws.org/

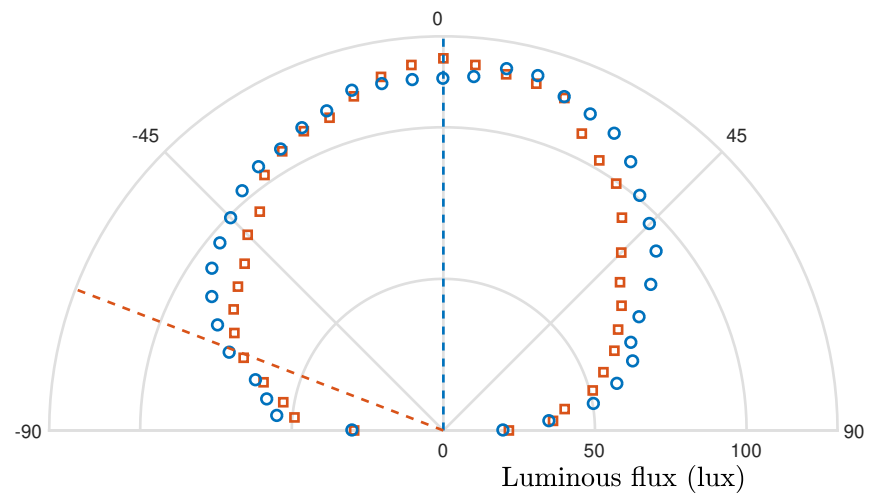

Fig S3. Luminous flux of diffuse sunlight as a function of the orientation with respect to the zenith. Data collected at a Latitude of $45.76^{\circ}$ North) in overcast weather on December 6, 2017, at the solar noon (12:45 AM UTC+1). Red squares: East $\left(-90^{\circ}\right)$-West $\left(+90^{\circ}\right)$ scan, blue circles: South $\left(-90^{\circ}\right)-$ North $\left(+90^{\circ}\right)$ scan. The dashed lines indicate the position of the sun $\left(21^{\circ}\right.$ above the horizon, directly south). The data illustrate that the incoming flux is maximum around the vertical direction although the sun in much closer to the horizon than to the zenith. The luminous flux is not ideally isotropic by varies only slightly in a region of $\pm 45^{\circ}$ around the zenith.
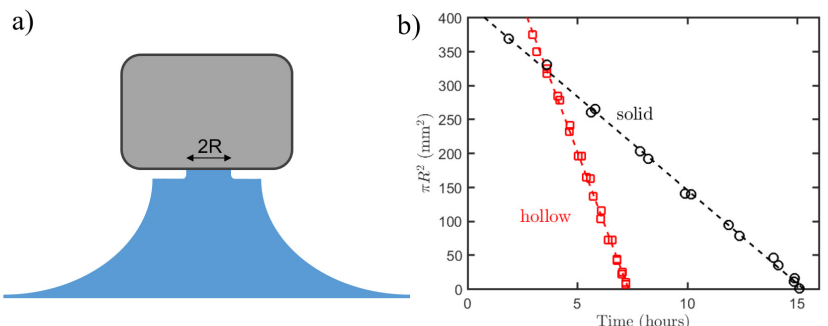

Fig S4. IR absorption and rapid sublimation in the lyophilizer. Objects made of material that absorbs infrared wavelengths (sand-blasted metals or most natural rocks) rather than reflecting them, slightly heat up during the formation of the pedestal, causing the ice in direct contact with the object to sublimate faster. A second small (less than $1 \mathrm{~mm}$ in thickness) foot (see a), of radius $\mathrm{R}$, appears and rapidly (within hours) sublimates, cause the object to prematurely fall off. (b) The surface of that secondary foot, computed from the measurement of $\mathrm{R}$ decreases linearly in time which indicates that the conductive flux from the stone to the ice remains constant. The rate at which this disturbing regime occurs is considerably larger for a hollow cylinder (red square) than for a solid one of identical size (black circles) due to the lower thermal capacity of the object. In Nature, a much more efficient energy balance is ensured through thermal exchanges with the surrounding atmosphere but the secondary foot can occasionally be observed (see Fig. 1a). 